\title{
The role of physicians in advocating for a national strategy for suicide prevention
}

\author{
Paul S. Links MD
}

See also research article by Katz and colleagues on page 1977 and at www.cmaj.ca/lookup/doi/10.1503/cmaj.110749

$\mathrm{T}$ he burden of suicide in Canada remains high. In 2007, 3611 individuals died by suicide $^{1}$ - about 10 people every day. The age-standardized mortality for suicide that year was 10.2 per 100000 population, which was higher than the age-standardized mortality for renal failure (7.9 per 100000$)$ and chronic liver disease and cirrhosis (6.4 per 100000$){ }^{2}$ Although suicide has been studied for more than 100 years, much of the progress in preventing suicide has been achieved in the last three decades. Several countries have adopted national strategies for suicide prevention; these countries include Australia, Denmark, England, Estonia, Finland, Germany, Greenland, Japan, Ireland, Northern Ireland, the Netherlands, New Zealand, Norway, Scotland, Sri Lanka, Sweden, the United States and Wales. ${ }^{3}$

Canada does not have a national strategy for suicide prevention despite specific guidance for one from the Canadian Association for Suicide Prevention in its 2004 blueprint (updated in 2009). ${ }^{3}$ Ironically, the objectives of most existing strategies around the world for the prevention of suicide are based on expert consensus guidelines that were formulated at a meeting in Calgary and Banff, Alberta, and adopted by the United Nations in $1996 .{ }^{3}$ Common themes in these strategies include public education, responsible media reporting, school-based programs, detection and treatment of depression and other mental disorders, attention to those with alcohol and drug abuse, attention to those with somatic illness, enhanced access to mental health services, improvement in the assessment of attempted suicide, postvention, crisis intervention, work and unemployment policy, training and education of health professionals, and reduced access to lethal methods of suicide. ${ }^{4}$

Many Canadian physicians, policy-makers and politicians have not been adequately updated by experts in the field that suicide is preventable. As a result, physicians in particular may not be aware of their important role in suicide prevention. In this article, I examine the arguments for and against the need for a national strategy for suicide prevention and selectively review some of the evidence of effective strategies that may influence physicians' practices.

\section{The evidence}

Some may argue that a move to develop a national strategy is premature. It is true that many questions remain about the causes and prevention of suicide and suicidal behaviour. Progress in preventing suicide is always problematic because suicidal phenomena are complex, suicide as an outcome is a rare event, suicide and mental illness are highly stigmatized and many true experiments related to suicidal individuals are not appropriate. Because the suicidal individual is no longer available for study, this field has to depend on surrogate outcomes, and the robustness of these outcomes, including suicide ideation, have not been adequately shown. In spite of these limitations, John Mann and colleagues ${ }^{5}$ systematically reviewed recent progress in establishing evidence-based prevention strategies for suicide. They developed estimates of the impact of different interventions on national rates of suicide; for example, education of primary care physicians led to a $22 \%-73 \%$ decline in the annual rate, and restricting access to means led to a $1.5 \%-33 \%$ decrease. ${ }^{5}$

At least three national strategies for suicide prevention have undergone systematic evaluations. The Finnish National Suicide Prevention

\section{KEY POINTS}

- In 2007 in Canada, 3611 individuals died by suicide - about 10 people every day.

- Much progress in preventing suicide has been achieved in the last three decades.

- Many developed countries have adopted national strategies to prevent suicide; Canada has not.

- Physicians can play an important role in preventing suicide, including providing collaborative care with mental health providers and improving community linkage after hospital stays.
Competing interests: Paul Links has received an unrestricted educational grant from Eli Lilly Canada.

This article has been peer reviewed.

Correspondence to:

Dr. Paul Links, paul.links@utoronto.ca

CMAJ 2011. DOI:10.1503 /cmaj.111008 
Program, which began in 1985, has been shown to be largely successful, particularly in involving a large number of professionals from various sectors in suicide prevention. ${ }^{6}$ The rate of suicide in Finland decreased 9\% from the 1985 level. ${ }^{7}$ A recent study of progress of Australia's National Suicide Prevention Strategy, which began in 1999, showed a reduction in the suicide rate between 1999 and 2004 from 22 to 17 per 100000 among men and from 5 to 4 per 100000 among women. Although the authors described the strategy as well regarded, they called for a reformulation of the program toward more selective and indicated interventions in the future. ${ }^{8}$ Prevention strategies can be conceptualized based on "who" is the target population: universal meaning the general population, selective referring to high-risk subgroups within the total population and indicated addressing those individuals at high-risk for suicide.

Scotland is also systematically evaluating its Choose Life strategy for suicide prevention, which was launched in 2002. In the phase 2 evaluation, the program was shown to be successful in raising awareness of suicide, increasing the skills and capacity of the nation by training more than 10000 people in suicide awareness and intervention skills, improving the knowledge and evidence base for suicide prevention, and bettering the reporting of suicide in the media. ${ }^{9}$ Although the initial objectives of this strategy called for a $20 \%$ reduction in suicide, the phase 2 evaluation suggested that a lower or directional (nonquantitative) target was more realistic. ${ }^{9}$

\section{Establishing a strategy in Canada}

The Canadian federal government is perfectly placed to proceed on the issue of suicide prevention. Universal health care allows the federal government to reach the most vulnerable individuals, and Statistics Canada has the capacity to develop an excellent surveillance system to monitor highrisk and selected groups and suicides. The federal government, through the Canadian Institutes of Health Research, can target initiatives in suiciderelated research. In addition, the federal government can modify access to means (e.g., gun control legislation) and take leadership in health promotion initiatives such as the initiative through the Mental Health Commission of Canada to target stigma. In addition, the federal government has primary responsibility of health care for highrisk populations such as First Nations people, prison populations and military personnel.

Although some provinces, including British Columbia, Alberta, Manitoba, New Brunswick and Nova Scotia have undertaken strategies for suicide prevention, and Nunavut and Ontario are working toward such strategies, the federal government must provide the central organizing and coordinating role for a national strategy to be successful. With the federal-provincial health accord up for renewal in 2014, there is an increased desire for accountability in health care spending. It may be appropriate to set targets such as reductions in suicide rates as quality indicators of improved mental health care. The federal government, which could operate in tandem with the Mental Health Commission of Canada's Mental Health Strategy for Canada, should ensure that a coordinating body has the resources and mandate to launch the strategy and should insist that an overarching approach for evaluation, such as the one used in Scotland ${ }^{10}$ is built into the strategy.

On Nov. 5, 2010, Megan Leslie, the sitting NDP MP for Halifax introduced Private Member's Bill C-593, directing the federal government to establish a national strategy for suicide prevention, in consultation with the provincial, territorial and First Nations governments. It is anticipated that this private members bill will be reintroduced in the fall 2011 Parliamentary session.

\section{Physician's role}

Physicians should be aware of the progress in developing effective strategies for suicide prevention that may directly affect the development of clinical services or patient care. In selecting recent research to highlight, I searched MEDLINE using the search terms "suicide prevention" and "national strategy" and looked for papers published since the review by Mann and colleagues in $2005 .{ }^{5}$ I selected only articles that appeared to be directly relevant to physicians, including the education of primary care physicians and collaborative care, improved community linkages after hospital discharge, and treatment interventions for individuals with psychiatric disorders at high risk of suicide behaviour.

\section{Education and collaborative care}

Collaborative models for treating depression in adults aged 60 years and older that link primary care providers with mental health specialists are particularly promising interventions to reduce suicide ideation and depression in this group. Suicidal older adults typically present to a medical provider shortly before attempting suicide. ${ }^{11}$ Findings of the Prevention of Suicide in Primary Care Elderly Collaborative Trial (PROSPECT), a multi-site study involving depressed adults aged 
60 years or older recruited through primary care practices, further showed that collaborative models of primary and mental health care might effectively reduce depression and suicide ideation among older adults. ${ }^{12}$ Patients were randomly assigned to a clinical algorithm consisting of antidepressants (primarily a selective serotonin reuptake inhibitor) and/or psychotherapy delivered by depression case managers or to augmented usual care. There was a greater decrease in the prevalence of suicide ideation at four months follow up among those receiving the intervention (from $29.4 \%$ to $16.5 \%$ ) than among those receiving usual care (from $20.1 \%$ to $17.1 \%)(p=0.01) .{ }^{12}$ Subsequently, the PROSPECT investigators found a 2.2 times greater decline from baseline in the rate of suicide ideation in the intervention versus the control group. The intervention group had a lower severity of depressive symptoms and a higher response rate over the 24-month follow-up compared with patients receiving usual care. ${ }^{13}$

\section{Improving community linkages after hospital discharge}

Patients recently discharged from acute psychiatric inpatient settings are at high risk of suicide. This increased risk in the postdischarge period has been observed in different countries and populations and for different periods. ${ }^{14} \mathrm{~A}$ recent Canadian study involving high-risk patients with a lifetime history of suicidal behaviour and current suicidal ideation confirmed the significant risk in the postdischarge period. Suicide and suicidal behaviour were common in this cohort, with 4 of $120(3.3 \%, 95 \%$ CI $0.9 \%-8.3 \%)$ participants dying by suicide and 41 of 104 (39.4\%, 95\% CI 30.0\%-49.5\%) reporting suicidal behaviour in the six months after discharge. ${ }^{15}$ Knesper recently summarized the evidence for rapid follow-up after discharge from emergency departments or inpatient settings and concluded that early contact after discharge led to significant reductions in suicide reattempts compared with usual care. ${ }^{16}$ Attempts to improve community linkages after discharge appear to be simple but effective ways of preventing repeated suicidal behaviour and, perhaps, suicides. ${ }^{16}$

\section{Interventions for people with psychiatric disorders at high risk of suicide}

In terms of pharmacologic management of the risk of suicide in patients with bipolar disorder, and likely other depressive disorders, evidence from a systematic review and meta-analysis of existing trials and a randomized controlled trial support the role of lithium as the treatment of choice for those at high risk of suicide. ${ }^{17}$ The
INTERSEPT (International Suicide Prevention Trial) trial provided an indication for clozapine in patients with schizophrenia or schizoaffective disorder who were deemed at high risk of suicide. ${ }^{17}$ For patients with borderline personality disorder and recurrent suicidal behaviour, at least half a dozen individual psychosocial treatments have been shown to be efficacious based on randomized controlled trials. In particular, dialectical behavioural therapy, a specific cognitive behavioural therapy that targets suicidal behaviour in patients with borderline personality disorder, has been shown to decrease the risk of future suicide attempts, lessen the medical risk from subsequent suicidal behaviour and decrease the likelihood of emergency department visits for suicidal behaviour. ${ }^{18}$ In a recent Canadian trial, outpatient psychiatric treatment provided by clinicians experienced in the care of patients with borderline personality disorder and recurrent suicidal behaviour showed that such an approach was efficacious after one year of treatment and over two years of follow-up. ${ }^{18}$

These findings have led experts to extract a limited number of principles of psychotherapy that may be effective in reducing the risk of future suicidal behaviour. Similar attempts to derive basic principles were undertaken in a careful review of the treatment manuals of psychotherapy for patients with borderline personality disorder ${ }^{19}$ and from a consensus statement developed by the US-based Group for the Advancement of Psychiatry Psychotherapy Committee. ${ }^{20}$ The basic principles of management are outlined in Box 1.

\section{Advocacy}

Certain universal strategies for prevention that have been described as "most promising" by Mann and colleagues 5 include following media guidelines for the reporting of suicides and suicidal behaviour and restricting access to means of suicide.

One of the most acknowledged natural experiments in suicide prevention was the decrease in

Box 1: Principles of psychotherapy that may be effective in reducing the risk of future suicidal behaviour

- Adopt a theoretical model of understanding suicidal behaviour to promote the confidence and understanding to work with suicidal patients

- Provide a stable treatment framework by increased activity of the therapist

- Convey empathic validation and the need for the patient to control his or her destructive behaviour

- Foster a greater sense of self-agency through the therapeutic relationship

- Establish a connection between destructive behaviours and feelings

- Develop methods with the patient to help him or her differentiate between nonlethal and "true" suicidal intention

- Set a low threshold for seeking consultation or supervision 
media coverage of subway-related suicides in Austria that led to an $80 \%$ decrease in such suicides. $^{21}$ The Toronto Transit Commission documented that, after creating a media reporting protocol to restrict the coverage of individual suicides in 1971, the rate of suicide incidents (suicides and attempts) never returned to the level experienced in 1971, except in one year (John O’Grady, Toronto Transit Commission Chief Safety Officer; personal communication, May 30, 2011). Guidelines on media reporting appear to have a great impact; however, systematic studies of such guidelines are still needed to validate these natural experiments.

Reducing access to lethal means is another promising intervention for preventing suicide. In developed nations, this can include passing legislation to reduce access to firearms, reducing carbon monoxide emissions from vehicles, installing barriers at bridges or other jumping sites, and restricting access to prescription medications. $^{22}$ In developing countries, ingestion of pesticides is estimated to be responsible for between 250000 and 300000 deaths per year and is a major means of suicide in countries such as China and Sri Lanka. ${ }^{23}$ Lockboxes for pesticides have been proposed as an intervention to reduce access to means of suicide. Hawton and colleagues $^{23}$ reported uptake of these devices by more than $80 \%$ of households in rural Sri Lanka.

Physicians can play a lead role in advocating for legislation that can reduce access to means of suicide.

\section{Conclusion}

Given the number of Canadians who die by suicide each year, the burden in terms of the suffering and pain of those left to cope with the loss of a loved one and the growing evidence of effective strategies for prevention, physicians have a responsibility to encourage governments to move toward policies and programs that will prevent suicides. In Canada, this includes encouraging the federal government to form a national strategy for suicide prevention similar to those in place in so many other developed nations. Physician can also reduce the risk of suicide by participating in collaborative care models for elderly patients with depression, improving community linkages for patients after discharge from inpatient psychiatric facilities and using target evidence-based management of patients at high-risk for suicide.

\section{References}

1. Suicide and suicide rate, by sex and by age group. Ottawa (ON): Statistics Canada. Available: www40.statcan.ca/101/cst01 /hlth66a-eng.htm (accessed 2011 May 25).

2. Age-standardized mortality rates by selected causes, by sex. Ottawa (ON): Statistics Canada. Available: www40.statcan.ca/ 101/cst01/health30a-eng.htm (accessed 2011 Sept. 7).

3. The CASP National Suicide Prevention Strategy. Winnipeg (MB): The Canadian Association for Suicide Prevention; 2009. Available: www.suicideprevention.ca (accessed 2011 May 26).

4. Anderson M, Jenkins R. The national suicide prevention strategy for England: the reality of a national strategy for the nursing profession. J Psychiatr Ment Health Nurs 2006;13:641-50.

5. Mann JJ, Apter A, Bertolote J, et al. Suicide prevention strategies: a systematic review. JAMA 2005;294:2064-74.

6. Hakanen J, Upanne M. Evaluation strategy for Finland's suicide prevention project. Crisis 1996;17:167-74.

7. Kerkhof AJFM. The Finnish national suicide prevention program evaluated. Crisis 1999;20:50,63.

8. Robinson J, McGorry P, Harris MG, et al. Australia's national suicide prevention strategy: the next chapter. Aust Health Rev 2006; 30:271-6.

9. Choose Life: the national strategy and action plan to prevent suicide in Scotland. Research, evidence and evaluation. NHS Health Scotland; 2011. Available: www.chooselife.net/Evidence /researchandevaluation.aspx (accessed 2011 May 26).

10. Mackenzie M, Blamey A, Halliday E, et al. Measuring the tail of the dog that doesn't bark in the night: the case of the national evaluation of Choose Life (the national strategy and action plan to prevent suicide in Scotland). BMC Public Health 2007;7:146-53.

11. Heisel MJ, Links PS. Primary care prevention of suicide among older adults. Geriatr Aging 2005;8:36-41.

12. Bruce ML, Ten Have TR, Reynolds CF, et al. Reducing suicidal ideation and depressives symptoms in depressed older primary care patients. A randomized controlled trial. JAMA 2004;291:1081-91.

13. Alexopoulos GS, Reynolds GF, Bruce ML, et al. Reducing suicidal ideation and depression in older primary care patients: 24-month outcomes of the PROSPECT study. Am J Psychiatry 2009;166: 882-90.

14. Troister T, Links P, Cutcliffe JR. Review of predictors of suicide within 1 year of discharge from a psychiatric hospital. Curr Psychiatry Rep 2008;10:60-5.

15. Links PS, Nisenbaum R, Ambreen M, et al. Prospective study of risk factors for increased suicide ideation and behavior following recent discharge. Gen Hosp Psychiatry. In press.

16. Knesper DJ. Continuity of care for suicide prevention and research: suicide attempts and suicide deaths subsequent to discharge from the emergency department or psychiatric inpatient unit. Newton (MA): American Association of Suicidology and the Suicide Prevention Resource Centre, Education Development Center; 2010. Available: www.sprc.org/library/continuityofcare .pdf (accessed 2011 Aug. 3).

17. Saunders KEA, Hawton K. The role of psychopharmacology in suicide prevention. Epidemiol Psichiatr Soc 2009;18:172-8.

18. McMain SF, Links PS, Gnam WH, et al. A randomized controlled trial of dialectical behavior therapy versus general psychiatry management for borderline personality disorder. Am J Psychiatry 2009;166:1365-74.

19. Weinberg I, Ronningstam E, Goldblatt MJ, et al. Strategies in treatment of suicidality: identification of common and treatmentspecific interventions in empirically supported treatment manuals. J Clin Psychiatry 2010;71:699-706.

20. Circular letter \#608. Post-meeting winter January 2009. Dallas (TX): Group for the Advancement of Psychiatry; 2009. Available: http://ourgap.org/ (assessed 2011 May 30).

21. Etzersdorfer E, Sonneck G. Preventing suicide by influencing mass-media reporting: the Viennese experience 1980-1996. Arch Suicide Res 1998;4:67-74.

22. Beautrais AL. National strategies for the reduction and prevention of suicide. Crisis 2005;26:1-3.

23. Hawton K, Ratnayeke L, Simkin S, et al. Evaluation of acceptability and use of lockable storage devices for pesticides in $\mathrm{Sr} i$ Lanka that might assist in prevention of self-poisoning. BMC Public Health 2009;9:69-80.

Affiliation: Paul Links is with the Department of Psychiatry, University of Toronto, Toronto, Ont. 